\title{
Genetic Basis of Giant Embryo Traits and Effects of Environmental Factors on Giant Embryo Rice
}

\author{
Bo Peng ${ }^{1}$, Kun $\mathrm{Xu}^{1}$, Kun $\mathrm{He}^{1}$, Dong-Ying Tang ${ }^{1}$, Juan Peng ${ }^{2}$, Xia-Yu Tian ${ }^{1}$, Yan-Fang Sun ${ }^{1}$, Xiao-Hua Song ${ }^{3}$, \\ Lu-Lu He ${ }^{1}$, Rui-Hua Pang ${ }^{1}$, Jin-Tao Li ${ }^{1}$, Quan-Xiu Wang ${ }^{1}$, Wei Zhou ${ }^{1}$, Hui-Long Li ${ }^{3}$, Hong-Yu Yuan ${ }^{1} \&$ \\ Xin-Xiang $\mathrm{A}^{4}$ \\ ${ }^{1}$ College of Life Sciences and Institute for Conservation and Utilization of Agro-bioresources in Dabie \\ Mountains, Xinyang Normal University, Xinyang, China \\ ${ }^{2}$ Xinyang Station of Plant Protection and Inspection, Xinyang, China \\ ${ }^{3}$ Xinyang Academy of Agricultural Science, Xinyang, China \\ ${ }^{4}$ Yunnan Provincial Key Lab of Agricultural Biotechnology of Yunnan Academy of Agricultural Sciences \& \\ Scientific Observation Station for Rice Resource of Yunnan, Ministry of Agriculture, Yunnnan Kunming, China \\ Correspondence: Bo Peng, College of Life Sciences and Institute for Conservation and Utilization of \\ Agro-bioresources in Dabie Mountains, Xinyang Normal University, Xinyang 464000, China. E-mail: \\ pengbo@xynu.edu.cn \\ Xin-Xiang A, Yunnan Provincial Key Lab of Agricultural Biotechnology of Yunnan Academy of Agricultural \\ Sciences \& Scientific Observation Station for Rice Resource of Yunnan, Ministry of Agriculture, Yunnnan \\ Kunming 650223, China. E-mail: ahope2002@163.com
}

Received: September 27, 2019

Accepted: October 22, 2019

Online Published: November 1, 2019

doi:10.5539/jmbr.v9n1p149

URL: https://doi.org/10.5539/jmbr.v9n1p149

\begin{abstract}
Giant embryo rice (GMR) is a special rice which can produce eutrophic functional rice. Giant embryo rice and its intensive processing products have been widely used in food, medicine, health care products and other fields, with extremely important scientific significance and economic value. In recent years, a series of important advances have been made in the research of giant embryo rice, and its achievements have attracted the attention of rice breeders and consumers at home and abroad. In this paper, the genetic basis of giant embryo traits and the effects of environmental factors on giant embryo rice were reviewed and analyzed, and the application prospect of giant embryo rice was also prospected, these will provide important reference for genetic improvement and application promotion of giant embryo rice.
\end{abstract}

Keywords: Giant Embryo Rice, Genetic Basis, Environmental Factors, Traits

\section{Introduction}

Rice (Oryza sativa L.) is one of the main grain crops in China. Rice production accounts for about $40 \%$ of the total grain production. More than two-thirds of the people live on rice in China (Li et al., 2018b; Peng et al., 2018 b). With the continuous improvement of people's living standards, high-quality rice is more and more popular with consumers (Zhou et al., 2014; Peng et al., 2014b). However, rice quality is a relatively complex quantitative trait. It is generally believed that rice quality can be divided into four aspects: appearance quality, nutritional quality, grinding quality, cooking and eating quality (Zhang et al., 2007; Peng et al., 2018a). The quality traits of rice are mainly controlled by genes. At present, a number of QTLs affecting rice quality traits have been identified in rice (Peng et al., 2014a). The important functional genes were isolated, cloned and analyzed (She et al., 2010; Zhang et al., 2016; Wang et al., 2010; Li et al., 2014; Wang et al., 2015). Rice quality traits are also affected by environmental factors, such as soil conditions, temperature, day and night temperature difference and sunshine (Ambardekar et al., 2011; Li et al., 2018a). Among these environmental factors, climatic conditions have the most obvious effect on rice quality traits.

Giant embryo rice is a kind of rice which can produce high nutritional functional rice in special rice. Compared with ordinary rice, the embryo of giant embryo rice increases about 2-3 times. The content of protein, mineral and vitamin is higher than that of ordinary rice. It has higher antioxidant activity and better nutritional and health functions. In addition, large amounts of oryzanol and $\gamma$-Aminobutyric acid are found in giant embryo rice, which 
have anti-cancer and anti- hyperlipidemia functions (Zhang et al., 2005; Seo et al., 2011; Fitzgerald et al., 2003; Kang et al., 2011). Because giant embryo rice is consistent with people's current food consumption concept and healthy life concept, it has become one of the most competitive nutritive rice in the future, which has attracted the attention of rice genetic and breeding experts at home and abroad in recent years. Over the past decade, many research groups at home and abroad have conducted in-depth studies on nutrition and quality traits, important agronomic traits, intensive processing of giant embryo rice and efficient utilization of byproducts, and made a series of important new progress (Rathore et al., 2018; Coleman et al., 2018; Fitzgerald et al., 2003; Kang et al., 2011). Particularly noteworthy is that in the application of giant embryo rice, the new varieties bred have been initially demonstrated and popularized in a large area, and some products related to intensive processing have been widely used in many fields such as health care, food, medicine and so on. Especially in the field of medical treatment and health care, the processing products of giant embryo rice are playing an increasingly important role, which shows that the products related to the intensive processing of giant embryo rice have important value and broad application prospects. In this paper, the genetic basis of giant embryo traits and the effects of environmental factors on mega-embryo rice were reviewed and analyzed, and the application prospect of giant embryo rice was prospected, in order to provide reference for genetic improvement and application promotion of giant embryo rice.

\section{Genetic Basis of Giant Embryo Traits}

\subsection{Study on the Localization of Giant Embryo Genes}

Japanese rice breeders used chemical mutagen N-methyl-N-nitrosourea (MUN) to treat the seed cells of rice variety Kinmaze, and then obtained a giant embryo mutant rice (Satoh et al., 1981). Through in-depth genetic analysis, the gene controlling giant embryo was located on chromosome 7 of rice, which is called giant embryo gene (Ge) (Satoh et al., 1990). Korean rice breeders also used N-methyl-N-nitrosourea chemical mutagen to treat the fertilized immature embryos of Japonica rice Hwacheongbyeo, induced a series of giant embryo mutant rice, and obtained the germplasm of giant embryo Japonica rice (Kim et al., 1991). Molecular mapping showed that the genes controlling giant embryo were also located at the long arm end of chromosome 7 (Satoh et al., 1981; Koh et al., 1996), which was the same allele as the giant embryo gene Ge (Kim et al., 1992). Using F2 population constructed by Hwacheongbyeo-ges and Milyang23, the giant embryo gene was located between two molecular markers RZ395 and CD0497 on chromosome 7 (Koh et al., 1996) with genetic distances of $2.4 \mathrm{~cm}$ and $3.4 \mathrm{~cm}$ (Ping et al., 2015). The genetic distances with microsatellite markers RM10 and RM18 were $9.6 \mathrm{~cm}$ and $7.7 \mathrm{~cm}$ respectively (Zhang et al., 2010). The F2 population constructed by crossing Kinmaze giant embryo mutant of Japonica Rice with Nanjing 11 of indica rice was studied. The results showed that the giant embryo gene was closely linked to RG678 molecular marker on chromosome 7. The genetic distance between them was $6.2 \mathrm{~cm}$ (Qian et al., 1996). The genetic distance between giant embryo gene and another molecular marker RZ395 on chromosome is $13 \mathrm{~cm}$. A large number of giant embryo rice varieties, such as Haiminori, Mebaemochi, Koiazusa and Haiibuki, have been bred by crossing Kinmaze giant embryo mutant EM40 with high-yielding variety Akenohoshi (Endo et al., 2006; Ishii et al., 2013; Matsushita et al., 2008; Maeda et al., 2005; Uehara et al., 2003). These giant embryo rice varieties are widely cultivated as functional rice.

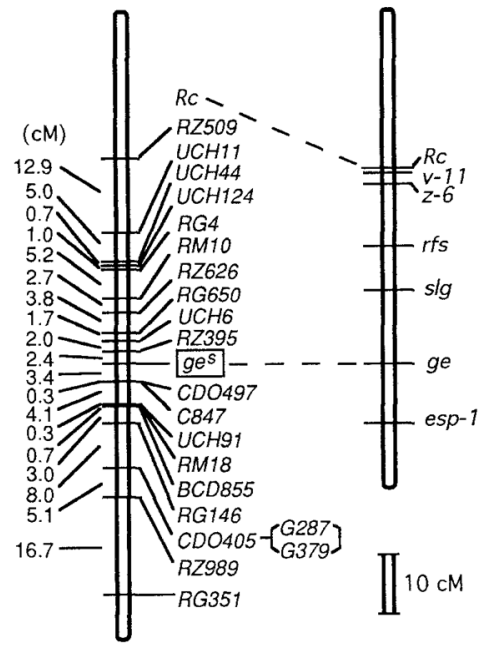

Figure 1. Linkage map of the segment of rice chromosome 7 aroundthe ge locus

Molecular map developed during this study on left; markers on the framework map are located with LOD > 3.0; geneticdistances in Kosambi (1944) units. Classical linkage map on right (Kinoshita et al., 1993). 
Further genetic analysis showed that the giant embryo traits of giant embryo rice were controlled by a single recessive gene. The giant embryo gene $(G E)$ was initially located between RG678 and RZ395 markers on chromosome 7 of rice, and the genetic distances were $6.2 \mathrm{~cm}$ and $13.0 \mathrm{~cm}$ respectively (Ping et al., 2015). The genetic analysis of giant embryo mutant in indica rice also showed that giant embryo was inherited by recessive single gene, and the size of seed embryo was directly determined by the genotype of fertilized eggs. Therefore, giant embryo traits could be directly selected in cross breeding F2 or mutagenesis M2 (Li et al., 2004). Interestingly, the giant embryo traits of giant embryo rice $98-14 \mathrm{geB}$ and Mge86 were studied. The results showed that giant embryo was caused by single recessive gene mutation, and the giant embryo gene and Japonica giant embryo rice variety 1137 was different alleles of the same locus (Zhang et al., 2005; Zhang et al., 2007a). At present, most studies on the inheritance of giant embryo traits in giant embryo rice show that giant embryo traits is controlled by an invisible gene. The embryos of giant embryo rice were obviously larger than those of ordinary rice. The F1 hybrid of giant embryo rice 10217 and Nippon Qing showed normal embryos of Nippon Qing. In the F2 population of F1 self-crossing, normal embryos basically accorded with the separation ratio of 3:1 (Zhang, 2011). The results also showed that the characteristics of giant embryos were controlled by a pair of recessive genes. Rice not giant embryo traits are dominant to giant embryo traits. The giant embryo traits are controlled by the genotype of the seed itself, and are not affected by the maternal genotype (Zhang et al., 2007b). Some research groups believe that embryos, though not endosperm traits, belong to rice seed traits, and their size may be controlled by endosperm development-related genes (Hong et al, 1995). Because giant embryo gene is controlled by a pair of recessive genes, its traits is controlled by the genotype of the seed itself, but it has not been determined whether the size of the embryo is regulated by endosperm gene or embryo gene. At present, there are at least 12 reported giant embryo alleles, such as $g e^{m}, g e, g e^{t}, g e^{s}, g e^{c}$, which are alleles of giant embryo gene $(O s 07 g 0603700)$. Among the rice varieties planted in different regions, $G E$ alleles were found successively such as ge-1, ge-2, ge-3, ge-4, ge-5, ge-6, ge-7, ge-8, ge-9, ge-10, get and GE alleles in "10217" giant embryo rice and "Kita-ake" giant embryo rice. Therefore, $G E$ gene of giant embryo is rich in diversity among different giant embryo rice varieties. The results of preliminary mapping of QTL by multiple traits of DH population and parents showed that the size of rice mesoderm was controlled by multiple genes. Different from the results of classical genetic mapping, the genes determining the size of rice mesoderm are not located on chromosome 7 , but on chromosomes 3, 4 and 9 of rice, and also affect the embryo weight and embryo weight rate (Zhang et al., 2005). Therefore, giant embryo genes can be selected by hybridization or mutagenesis, and there are linkage reactions in giant embryo traits of the second generation. According to the recombination and exchange between individual plants in rice population, the location of giant embryo genes on chromosomes can be determined, which will provide a solid basis for molecular breeding of giant embryo genes.

\subsection{Molecular Breeding of Giant Embryo Gene}

According to the giant embryo gene ( $g e$ ), which has been isolated and cloned, and the molecular marker closely linked with giant embryo gene, giant embryo gene can be applied to the genetic breeding process of giant embryo rice. For example, giant embryo genes can be used for co-dominant molecular marker breeding, marker-assisted selection breeding, gene aggregation breeding and genome editing breeding to improve the genetics and molecular breeding of giant embryo rice. Through the detection and analysis of molecular markers closely linked to giant embryo genes, different genotypes can be distinguished, and the giant embryo genes can be directly used for molecular marker-assisted selection breeding or gene aggregation breeding. In the giant embryo gene of Shangshida No. 5, GE-2 marker can distinguish three different genotypes of GEGE, GEge and gege (Zhang et al., 2017), which can be directly used in the molecular marker-assisted selection breeding and gene aggregation breeding of ge gene, thus speeding up the breeding process of giant embryo rice new varieties. The giant embryo phenotype of giant embryo rice belongs to recessive trait, and involves double fertilization of triploid endosperm. Its genetic mechanism is relatively complex. It can't be observed in real time during the growth process (Zhang et al., 2017). Therefore, through the research and development of functional molecular markers of GEC giant embryo gene, we can improve the efficiency of giant embryo rice breeding and shorten the breeding cycle.

The cloned giant embryo gene can also be used as CAPS molecular marker for molecular breeding. According to the single nucleotide mutation at the coding chain +125 of the mega-embryo gene of Shangshida 5 , a new restriction site (Zhang et al., 2017) of Alu I (AGCT) was produced. On the basis of PCR amplification, the CAPS molecular marker of the amplified products of the giant embryo gene could be digested by restriction endonuclease, and then the method of detecting giant embryo gene mutants could be established. Using this functional molecular marker, genotypes with mutant sites of giant embryo gene can be screened accurately, which ensures the high accuracy of plant identification in the breeding process, thus laying an important 
foundation for the future research of breeding new giant embryo rice with "Shangshida 5" giant embryo rice as parents.

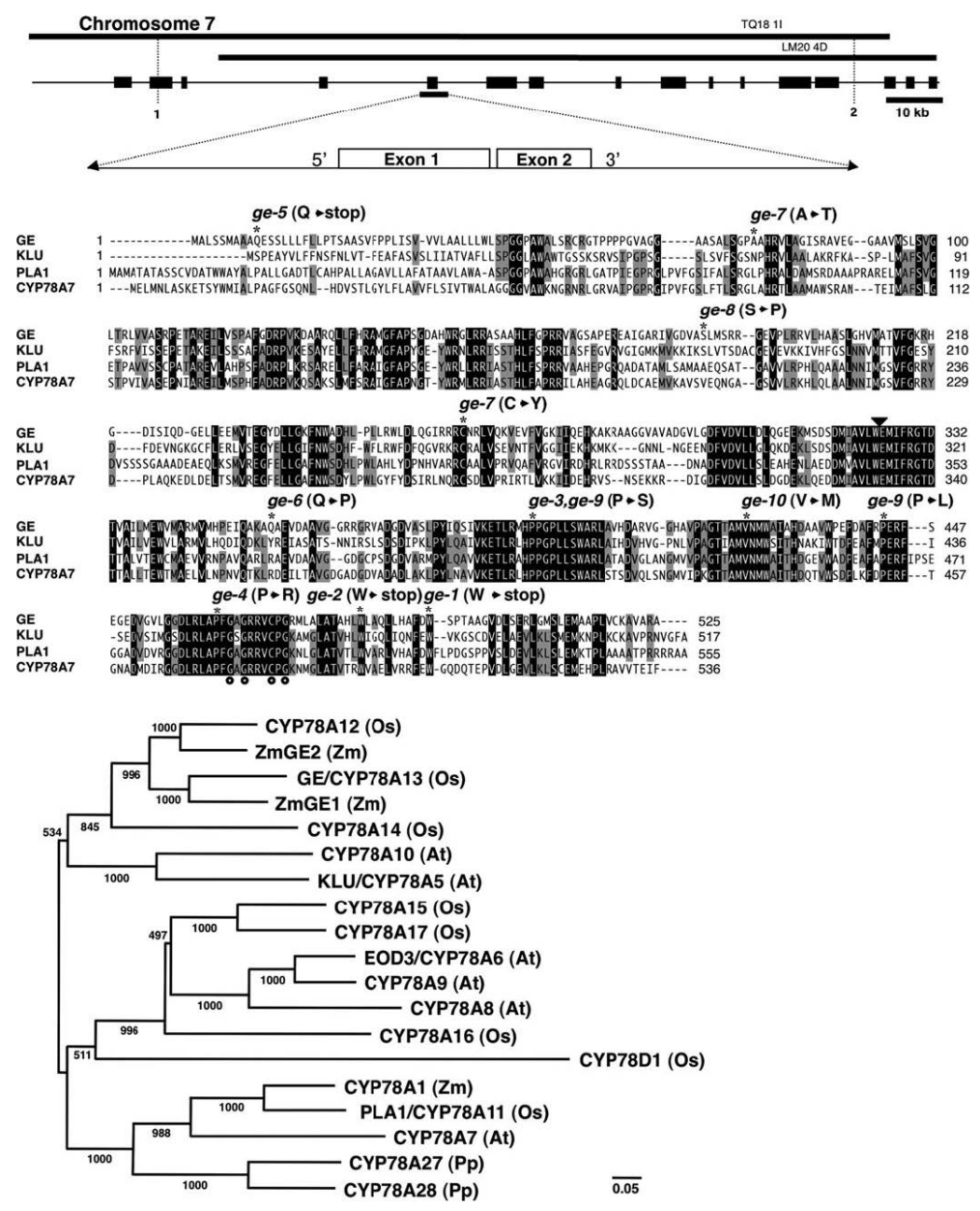

Figure 2. Molecular characterization of the GE gene (Nagasawa et al., 2013)

(a) The genomic region of the GE locus covered by two bacterial artificial chromosome (BAC) clones, TQ18-11 and LM20-4D. Filled boxes indicate the predicted open reading frames based on our BAC sequences. The numerals on both sides indicate the number of recombination break points. The complementing $5.1 \mathrm{~kb}$ EcoRI fragment is shown below, in which open boxes indicate two exons of the GE gene.

(b) Alignment of deduced amino acid sequences of four CYP78A family proteins (GE and PLA1 fromrice, KLU and CYP78A7 fromArabidopsis). Mutation sites of 10 ge alleles are indicated as asterisks. Residues conserved in all four proteins are shaded in black, and those conserved in three proteins are shaded in grey. The circled amino acids show the conserved residues at the heme-binding site (Chapple, 1998). The GE amino acid sequence of this figure (cv. Taichung 65) differs from that of Kinmaze (Figure S1), in which two non-conserved amino acids are substituted (R369G and (D439E).

(c) The phylogenetic tree of CYP78 family proteins from Oryza sativa (Os), Arabidopsis thaliana (At), Zea mays $(\mathrm{Zm})$ and Physcomitrella patens $(\mathrm{Pp})$ constructed by the neighbor-joining method. The bootstrap values based on 1000 replicates are shown as the numbers.

Some research results show that the yield of giant embryo rice will decrease. The genetic background of giant embryo rice bred by radiation mutagenesis was identical to that of the original variety. There was only one pair of recessive ge genes (Zhang et al., 2007c). The further results showed that ge gene had no significant effect on the main agronomic traits and some yield traits of hybrid rice, but ge gene could cause the decrease of 1000-grain weight, bulk density, theoretical yield and actual yield of hybrid rice, and had significant effect only when GE gene was homozygous. Molecular marker assisted breeding is a method of screening target genes by molecular marker technology derived from polymorphism among genes (Wang et al., 2015). In recent ten years, 
molecular marker assisted breeding technology has become more and more mature, which is closely combined with traditional hybrid breeding, fully demonstrating the superiority of molecular marker assisted breeding technology in rice genetic breeding.

Genome editing technology based on sequence-specific nucleases (SSNs) has become one of the most effective new tools for crop genetic improvement in recent years (Samanta et al., 2016; Liu et al., 2017). At present, various types of SSNs have been developed for genome editing, including zinc finger nucleases (ZFNs), transcription activator-like effector nucleases (TALENs) and the clustered regularly interspaced short palindromic repeat (CRISPR/Cas9) system, etc. (Noman et al., 2016; Gil-Humanes et al., 2017; Mao et al., 2013; Gaj et al., 2013). ZFNs, TALENs and CRISPR/Cas9 technologies are based on their own endonuclease activity, which causes DNA double strand breaks at the target DNA sequence location. Mutations are introduced by homologous recombination or non-homologous end-to-end connections. Then, targeted genome editing, such as gene knockout, insertion, point mutation, gene modification or transcriptional activation, can be successfully realized in different organisms. Genome editing technology based on SSNs has been widely used in basic research and applied research of plants. For example, using ZFNs, TALENs and CRISPR/Cas9 technologies, specific gene knockout, point mutation or gene modification (Liu et al., 2017) have been successfully implemented for many plants, including important crops such as rice, maize, wheat, barley, soybean and so on. (Li et al., 2017; Wendt et al., 2013; Haun et al., 2014; Liang et al., 2014; Wang et al., 2014; Shan et al., 2015). Among them, TALEN technology has been applied to rice genetic breeding (Shan et al., 2015; Birla et al., 2017), which provides a new strategy for rice breeders for genetic improvement and molecular breeding of giant embryo rice.

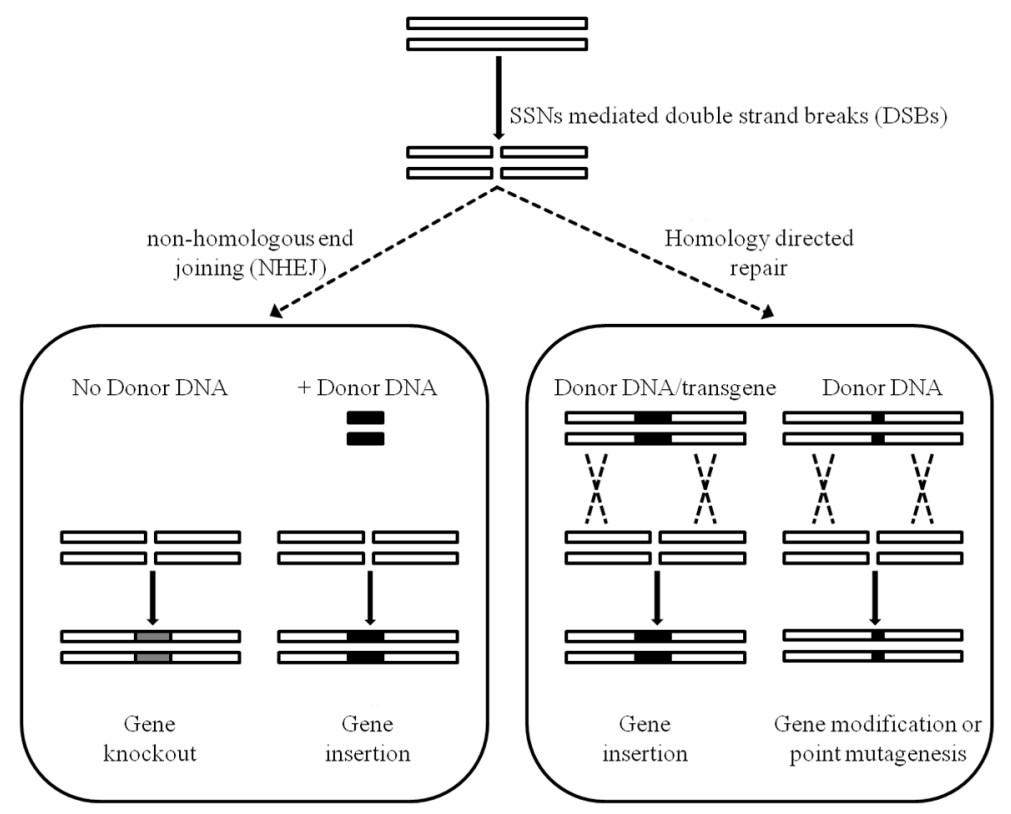

Figure 3. Overview of possible outcomes from genome editing based on sequence-specific nucleases (Peng et al., 2019)

\section{Effects of Environmental Factors on Giant Embryo Rice}

\subsection{Effects of Low Temperature Stress on Seed Germination of Giant Embryo Rice}

Among various environmental factors in nature, temperature plays an important role in the development of the rice, such as the germination, germination potential, germination rate and bud growth of the rice. Within a certain temperature range, with the decrease of temperature, the degree of Inhibiting Germination of giant embryo rice seeds increased, and the germination potential and germination rate decreased in varying degrees. At the same time, the decrease of germination potential and germination rate of giant embryo rice seeds under different low temperature stress was greater than that of common rice, that is, the germination of giant embryo rice seeds. Germination potential and germination rate were positively correlated with temperature (Zhang et al., 2014). The germination rate of giant embryo rice seeds decreased sharply when the stress temperature reached $8^{\circ} \mathrm{C}$ or below. 
The germination of giant embryo rice seeds could not recover well after low temperature treatment. The rate of dead seedlings was higher and the germination of seeds was hindered. After low temperature treatment at $6^{\circ} \mathrm{C}$ and below, the seedlings could not recover and eventually lead to dead seedlings of giant embryo rice seedlings (Zhang et al., 2014). In other words, the seeding death rate of giant embryo rice was negatively correlated with stress temperature.

Under the condition of low temperature stress, the bud length, coleoptile length and radicle length of giant embryo rice decreased with the decrease of stress temperature, and the difference between giant embryo rice and control reached a significant positive correlation level. Under different low temperature stress conditions, the sensitivity of different giant embryo rice low temperature stress is different. The variation range of bud length, coleoptile length and radicle length of common rice is smaller than that of giant embryo rice seeds, which indicates that giant embryo rice is more sensitive to low temperature stress at budding stage (Zhang et al., 2014). Because the environmental temperature has an important influence on the germination of giant embryo rice seeds and the growth of germination after germination, strict control of temperature can make giant embryo rice grow better in the actual production process.

\subsection{Effects of Water Stress on Seed Germination and Seedling Growth of Giant Embryo Rice}

There are two ways to culture giant embryo rice seeds under water stress. One is to soak them in PEG-6000 solution for 24 hour and then culture them with water. The second method is to soak giant embryo rice seeds in clear water for 24 hours and then culture them in PEG-6000 solution. The two different culture methods had different effects on the germination of the giant embryo rice seeds. Xi giant embryo 1, a new japonica type giant embryo rice line bred by Xichang university, was selected as the experimental material, and the conventional japonica rice hybrid line 22-2 (small embryo) widely promoted locally was used as the control. The rice was immersed in PEG solution of different concentrations for 24 hours, and then treated with clean water (Xiang et al., 2010). The results showed that PEG solution could promote the germination of giant embryo rice seeds to some extent, and the low concentration of PEG solution could promote the germination of giant embryo rice seeds more obviously. The concentration of PEG solution at $5 \%$ had the greatest effect on the germination of giant embryo rice seeds (Xiang et al., 2010); after soaking seeds in clear water for 24 hours, PEG solution with different concentrations was used to treat the seeds. This method had certain inhibitory effect on the germination of giant embryo rice seeds, and the higher the concentration was, the stronger the inhibitory effect was.

Different PEG stress modes had different effects on seed germination and seedling growth of giant embryo rice. The stress method of PEG liquid culture induced by clear water was not conducive to the germination and emergence of giant embryo rice seeds, and the seedling growth was irregular. With the increase of PEG concentration, the growth potential of giant embryo rice seedlings became weaker, the content of chlorophyll and soluble protein increased, the root activity decreased, and SOD activity increased under mild and moderate stress, and decreased under water drought stress. However, under the stress method of clear water culture induced by PEG liquid. Soluble protein content decreased, SOD activity and root activity increased. When PEG concentration was $15 \%$ or less, it promoted the germination, SOD activity, root activity, seedling emergence and seedling growth of giant embryo rice, but inhibited it when PEG concentration was higher than $15 \%$ (Dai ., 2012a; Dai ., 2012b). Therefore, the stress method of PEG induced clear water culture can make the giant embryo rice seeds germinate better and reach the optimal state of germination, which will lay a solid foundation for the faster and better growth of giant embryo rice.

\subsection{Effects of Cultivation and Management Measures on Quality and Yield of Giant Embryo Rice}

The formation of rice quality is mainly controlled by its own genes, but also by the environment and cultivation methods. Among them, the application amount of nitrogen fertilizer has the greatest influence on rice quality (Bao et al., 1954), and the effect of nitrogen fertilizer on rice quality is mainly manifested in the appearance quality. The results showed that the chalky rice rate and chalkiness of giant embryo rice decreased with the increase of nitrogen application, while the aspect ratio and transparency were less affected by nitrogen application. The protein content increased significantly with the increase of nitrogen application (Cong et al., 2017). The results were basically consistent with previous studies (Jin et al., 2001; Wan et al., 2006). Therefore, it is beneficial to improve the appearance quality of giant embryo rice by properly increasing the application of nitrogen fertilizer in a certain range.

In China, protein in rice is generally regarded as an important measurement index of rice nutritional quality, and the increase of protein content is often used as one of basis for improving rice quality. As for the nutritional quality of rice, with the increase of nitrogen application, the amylose content of rice will decrease, and the amylose content of giant embryo rice will also decrease. Studies have shown that within the range of 0-300 
$\mathrm{kg} \cdot \mathrm{Hm}^{-2}$ nitrogen application, the amylose content of giant embryo rice in different growth stages decreased with the increase of nitrogen fertilizer level, but the decrease extent of different giant embryo rice varieties was not the same (Gao et al., 2010). As for the effect of nitrogen fertilizer on protein content, the general results showed that the protein content in rice increased with the increase of nitrogen application (Lee et al, 2005; Dai et al., 2009). Interestingly, increasing nitrogen fertilizer in a certain range can effectively alleviate the negative impact of high temperature stress on rice quality and yield, and can promote the increase of protein content and improve the nutritional quality of rice. Therefore, in order to improve the nutritional quality and appearance quality of giant embryo rice, the amount of nitrogen fertilizer can be increased appropriately in a certain range. Rice shape (grain length, grain width, grain thickness and length-width ratio) is an important index of appearance quality traits of rice. Due to the lack of moisture in the soil, air temperature, nutrients, and other factors led to the early stage of the rice grain filling rate relatively quickly, and later premature aging, the synthesis and accumulation of starch affected, causing the arrangement of starch grains is not tight, chalk white area increases (Hong et al., 2011). Interestingly, because of the roundness and looseness of starch granules in the seeds of giant embryo rice, the grain hardness "Suweon 542" decreased significantly, and the grain hardness of dehulled grain was only $45 \%$ of that of the control variety, which indicated that "Suweon 542" was easy to be crushed during milling, and it was a rice variety suitable for dry milling (Mo et al., 2013; Altheid et al., 2012). This is expected to solve the high cost of wet grinding production, and cause water waste and soak process to cause environmental problems such as pollution. Therefore, in the production process of the giant embryo rice, it is suggested that the cultivation of the ridge is used to obtain excellent quality.

The number of grains per panicle and effective panicles per panicle of giant embryo rice are greatly affected by cultivation and management methods, that is, the cultivation and management of giant embryo rice is closely related to its yield. For example, the yield of giant embryo rice obtained by ridge cultivation is higher than that of submerged and dry cultivation. The effects of cultivation methods on the appearance, brown rice rate, protein content and amylose content of giant embryo rice were not obvious, but on the Chalky rate and chalk white area of rice. The panicle length and 1000-grain weight of rice are mainly related to the characteristics of rice varieties, and are less affected by cultivation methods, while the number of grains and effective panicles are more affected by cultivation methods. As a result, the yield of rice is affected by both varieties and cultivation methods (Dai et al., 2011). In the process of rice breeding, besides the characteristics of varieties themselves, we should pay attention to the study of high-quality and high-yield cultivation techniques to obtain higher yield.

\section{Prospect}

Giant embryo rice is a rice mutant with enlarged embryos, whose embryos account for $1 / 3$ to $1 / 4$ of the whole grain volume, more than twice the size of normal rice embryos. The contents of vitamins, amino acids, proteins, minerals, natural nutrients and physiological active substances in giant embryo brown rice were significantly higher than those in common brown rice. Especially, giant embryo brown rice was rich in gamma-aminobutyric acid (GABA) and vitamin E, which had multiple regulatory functions on human body. Therefore, brown rice which often eats giant embryo rice can regulate human physiological metabolism and prevent diseases, so giant embryo rice is known as "longevity rice" or "functional rice" by breeders.

At present, with the development of economy and society and the continuous improvement of living standards, people pay more and more attention to their own nutrition and health status. Green, nutritious and safe food is favored by the majority of consumers. Giant embryo rice is a "pure natural" green food with wide adaptability and high safety, which is more acceptable to consumers. Therefore, giant embryo rice, as a kind of functional rice with high nutrition, has broad prospects for development. In the future, giant embryo rice and its corresponding processing products will mainly concentrate on the following aspects: (1) Giant embryo rice: because giant embryo rice contains rich natural nutrients such as GABA, protein, minerals, amino acids, vitamins and so on, the nutritional value of giant embryo rice is higher than that of common ordinary rice. (2) Feed: giant embryo rice is rich in protein and fat, and its nutrient content is higher than that of ordinary rice. It is a good raw material for processing animal feed. (3) Rice oil: Rice embryo and rice bran contain a large amount of oil, rice bran oil contains $38 \%$ linoleic acid and $42 \%$ oleic acid, linoleic acid and oleic acid ratio is scientific and reasonable, has high nutritional value, has a higher nutritional and health function. The oil content of giant embryo rice is higher than that of common rice, and giant embryo rice can be used as raw material for extracting rice oil. (4) Health food: giant embryo rice contains abundant natural nutrients and physiological active substances such as protein, minerals, amino acids, vitamins and so on, especially GABA, which has the functions of brain-strengthening, retarding aging, improving kidney function, activating liver function and regulating human blood pressure. Energy-based food should be developed and utilized. It can be used as functional food. 
Although many new giant embryo rice varieties have been bred by rice breeders at home and abroad, their market share still needs to be improved. This is mainly due to the low transformation rate of the research results of giant embryo rice and the lack of market application and promotion; secondly, the high price of giant embryo rice can not be ignored as an important factor. However, with the continuous increase of population and market demand and the continuous emergence of deep processed products, many advantages of giant embryo rice will be further highlighted, and it will have broad application and promotion prospects.

\section{Acknowledgments}

This work was financially supported by National Natural Science Foundation of China (U1604110, U1404319, 31600992), Key Project of Science and Technology in Henan Province (192102110119, 182102110442), National Key R\&D Program Projects (2016YFD0100101-10), The Training Plan of Young Backbone Teachers in Colleges and universities of Henan Province (2019GGJS162), Postgraduate Education Reform Project of Henan Province (2019SJGLX088Y), Key Scientific Research Projects of Universities in Henan Province (19A180030), Nanhu Scholars Program for Young Scholars of XYNU (2016054), Scientific Research Innovation Project for Postgraduate of XYNU (2018KYJJ47).

\section{Conflict of interests}

The authors declare that there is no conflict of interests regarding the publication of this paper.

\section{References}

Altheide, M. C., Morawicki, R. O., \& Hager, T. J. (2012). Impact of milling and water-to-riceratio on cooked rice and wastewater properties. Food Science and Technology International, 18(3), 291-298.

Ambardekar et al. (2011). Impact of field-scale nighttime air temperatures during kernel development on rice milling quality. Field Crops Research, 122(3), 179-185.

Bao, W. K., \& Yan, J. R. (1954). Effects of fertilizer on crop growth and development. Journal of Agriculture, 2(3), 137-148.

Birla et al. (2017). Progress and challenges in improving the nutritional quality of rice (Oryza sativa L.). Critical Reviews in Food Science and Nutrition, 57(11), 2455-2481.

Coleman, G. L., \& Nelson, R. P. (2018). On the formation of compact planetary systems via concurrent core accretion and migration. Monthly Notices of the Royal Astronomical Society, 457(3), 2480-2500.

Cong et al. (2017). Effects of nitrogen fertilizer application rate on nitrogen use efficiency and grain yield and quality of different rice varieties. Chinese Journal of Applied Ecology, 28(4), 1219-1226.

Dai et al. (2009). Differences in response of rice quality to daytime warming under different nitrogen application water products. China Rice Science, 23(4), 421-426.

Dai et al. (2011). Effects of different cultivation methods on yield and quality of japonica giant embryo rice. China Agricultural Bulletin, 27(03), 179-183.

Dai, H. Y. (2012a). Effects of water stress on seed germination and seedling formation of japonica giant embryo rice. Journal of Southwest Agriculture, 25(1), 85-90.

Dai, H. Y. (2012b). Effects of water stress on seedling traits of japonica giant embryo rice. Journal of Yunnan Agricultural University, 27(4), 497-502.

Endo et al. (2006). Breeding of a new giant embryo rice cultivar "Koiazusa" with high tolerance to cool temperature. Bulletin of the National Agricultural Research Center for Tohoku Region, 105, 1-16.

Fitzgerald et al. (2003). A rheological and biological study. Journal of Agricultural and Food Chemistry, 51(8), 2295-2299.

Gaj et al. (2013). ZFN, TALEN, and CRISPR/Cas-based methods for genome engineering. Trends in Biotechnology, 31(7), 397-405.

Gao et al. (2010). Effects of nitrogen fertilizer levels on cooking and eating quality of rice with different growth types. Journal of China Academy of Science and Technology, 43(21), 4543-4552.

Gil-Humanes et al. (2017). High-efficiency gene targeting in hexaploid wheat using DNA replicons and CRISPR/Cas9. Plant Journal, 89(6), 1251-1262.

Haun et al. (2014). Improved soybean oil quality by targeted mutagenesis of the fatty acid desaturase 2 gene family. Plant Biotechnology Journal, 12, 934-940. 
Hong et al. (1995). Mutations affecting embryo size in rice. Rice Genetics Newsletter, 12, 196-199.

Ishii et al. (2013). "Haigokoro", a new rice cultivar with high-emergence rate, low-amylose content and giant embryo. Bulletin of the National Agricultural Research Center for Western Region, 12, 25-41.

Jin et al. (2001). Effects of nitrogen fertilizer on chalknessratio and cooking and eating quality properties of rice grain. Plant Nutrition and Fertilizer Science, 7(1), 31-35.

Kang et al. (2011). Physicochemical properties and eating qualities of milled rice from different Korean elite rice varieties. International Journal of Food Properties, 14(3), 640-653.

Kim et al. (1992). New mutants for endosperm and embryo characters in rice: Two dull endosperms and a giant embryo. Society for the Advancement of the Breeding Research in Asia and Oceania, 125-131.

Koh, H. J., Heu, M. H., \& Mccouh, S. R. (1996). Molecular mapping of the ges gene controlling the super-giant wmbryo character in rice. Theoretical and Applied Genetics, 93(1-2), 257-261.

Leesawatwong et al. (2005). Nitrogen fertilizer increases seed protein and milling quality of rice. Cereal Chemistry, 82, 588-593.

Li et al. (2004). Discovery and genetic breeding of giant embryonic indica rice. Journal of Crops, 30(2), $122-125$.

$\mathrm{Li}$ et al. (2014). Chalk5 encodes a vacuolar $\mathrm{H}+$-translocating pyrophosphatase influencing grain chalkiness in rice. Nature Genetics, 46, 389-404.

Li et al. (2017). High-efficiency breeding of early-maturing rice cultivars via CRISPR/Cas9-mediated genome editing. Journal of Genetics \& Genomics, 44(3), 175-178.

Li et al. (2018a). Effects of topdressing magnesium and zinc fertilizers at different periods on yield and quality of japonica rice with good eating quality. Scientia Agricultura Sinica, 51(8), 1448-1463.

Li et al. (2018b). Rice functional genomics research: Past decade and future. Molecular Plant, 11(3), 359-380.

Liang et al. (2014). Targeted mutagenesis in Zea mays using TALENs and the CRISPR/Cas system. Journal of Genetics and Genomics, 41, 63-68.

Liu et al. (2017). CRISPR-P 2.0: An improved CRISPR-Cas9 tool for genome editing in plants. Molecular Plant, (3), 530-532.

Maeda et al. (2005). A new rice variety with giant embryos, "Haiminori". Breeding Science, 51(3), 211-213.

Mao et al. (2013). Application of the CRISPR-Cas system for efficient genome engineering in plants. Molecular Plant, 6, 2008-2011.

Matsushita et al. (2008). A new rice cultivar with giant embryo, "Haiibuki". Bulletin of the National Agricultural Research Center for Western Region, (7), 1-14.

Mo et al. (2013). Agronomic and genetic analysis of Suweon 542, a rice floury mutant line suitable for dry milling. Rice, 6(1), 37.

Noman, A., Aqeel, M., \& He, S. (2016). CRISPR-Cas9: Tool for qualitative and quantitative plant genome editing. Frontiers in Plant Science, 7, 1740.

Peng et al. (2014a). Comparative mapping of chalkiness components in rice grain using five populations across two environments. BMC Genetics, 15, 49.

Peng et al. (2014b). OSAAP6 functions as an important regulator of grain protein content and nutritional quality in rice. Nature Communications, 5, 4847.

Peng et al. (2018a). A method for detection of main metabolites in aromatic rice seeds. Agricultural Biotechnology, 7(1), 112-116.

Peng et al. (2018b). The arrangement of endosperm cells and development of starch granules are associated with the occurrence of grain chalkiness in japonica varieties. Journal of Agricultural Science, 10(7), 156-166.

Peng et al. (2019). Genetic improvement of grain quality promoted by high and new technology in rice. Journal of Agricultural Science, 11(1), 81-94.

Ping et al. (2015). Molecular characterization, phenotype in-vestigation and expression analysis of a new giant embryo allele. Acta Botanica Boreali-Occidentalia Sinica, 35(8), 1518-1523.

Qian et al. (1996). Molecular mapping of rice giant embryo gene. Chinese Journal of Rice Science, 10(2), 65-70. 
Rathore, A., Gloviczki, P., \& Bjarnason, H. (2018). Management of giant embryonic vein in Klippel-Trénaunay syndrome. Journal of Vascular Surgery Venous \& Lymphatic Disorders, 6(4), 523-525.

Samanta, M. K., Dey, A., \& Gayen, S. (2016). CRISPR/Cas9: An advanced tool for editing plant genomes. Transgenic Research, 25(5), 561-573.

Satoh, H., \& Iwata, N. (1990). Linkage analysis in rice. On three mutant loci for endosperm properties, ge (giant embryo), du-4 (dull endosperm-4) and flo-1 (flouryendosperm-1). Japan J Breed, 40(2), 268-269.

Satoh, H., \& Omura, T. (1981). New endosperm mutations induced by chemical mutagens in rice (Oryza sativa L.). Japan J Breed, 31(3), 316-323.

Seo et al. (2011). Comparative analysis of physicochemicals and antioxidative properties of new giant embryo mutant, YR23517Acp79, in rice (Oryza sativa L.). Journal of the Korean Society for Applied Biological Chemistry, 54(5), 700-709.

Shan et al. (2015). Creation of fragrant rice by targeted knockout of the OsBADH2 gene using TALEN technology. Plant Biotechnology Journal, 13, 791-800.

She et al. (2010). A novel factor FLOURY ENDOSPERM2 is involved in regulation of rice grain size and starch quality. Plant Cell, 22, 3280-3294.

Uehara et al. (2003). A new rice variety "Mebaemochi". National Agricultural Research Center Cent., 2, 63-81.

Wan et al. (2006). Effect of nitrogen application on main quality and RVA profile characters of hybrid rice. Acta Agronomica Sinica, 32(10), 1491-1497.

Wang et al. (2010). Duplication and independent selection of cell-wall invertase genes GIF1 and OsCIN1 during rice evolution and domestication. BMC Evolutionary Biology, 10, 108.

Wang et al. (2014). Simultaneous editing of three homoeo-alleles in hexaploid bread wheat confers heritable resistance to powdery mildew. Nature Biotechnology, 32, 947-951.

Wang et al. (2015). Copy number variation at the GL7 locus contributes to grain size diversity in rice. Nature Genetics, 47, 944-948.

Wang, J., Wei, Y. L., \& Ji, X. L. (2015). Application of molecular marker technology. Shanghai Environmental Science Collection, (5), 227-230.

Wendt et al. (2013). TAL effector nucleases induce mutations at a pre-selected location in the genome of primary barley transformants. Plant Molecular Biology, 83, 279-285.

Xiang et al. (2010). Effects of water stress on germination and emergence of japonica giant embryo rice. Journal of Xichang University (Natural Science Edition), 24(2), 8-11.

Zhang et al. (2005a). Breeding and research of indica giant embryo male sterile line 98-14geA. Journal of Shanghai Jiaotong University (Agricultural Science Edition), 23(3), 223-228.

Zhang et al. (2005b). Comparative studies on major nutritional components of rice with a giant embryo and a normal embryo. Journal of Food Biochemistry, 29(6), 9.

Zhang et al. (2005c). QTL analysis of rice embryo weight-related traits. Journal of Crops, 31(2), 224-228.

Zhang et al. (2007a). Agronomic and yield characteristics of giant embryo hybrid rice. Journal of Tropical Crops, 28(2), 33-36.

Zhang et al. (2007b). Biological characteristics of giant embryo rice. Journal of Biology, 33(6), 1034-1037.

Zhang et al. (2007c). Mutagenic acquisition and genetic studies of Indica giant embryo germplasm. Chinese Agricultural Science, 40(7), 1309-1314.

Zhang et al. (2011). Identification of a new Ge allele in rice giant embryo Molecular marker development. Molecular Plant Breeding, 9(5), 525-530.

Zhang et al. (2016). Progresses in research on cloning and functional analysis of key genes involving in rice grain quality. Scientia Agricultura Sinica, 49(22), 4267-4283.

Zhang et al. (2017). Establishment of molecular markers for giant embryo gene of "Shangshi university 5" giant embryo rice. Molecular Plant Breeding, 15(11), 4512-4517.

Zhang, Q. F. (2007). Strategies for developing green super rice. Proceedings of the National Academy of Sciences of the United States of America, 104(42), 16402-16409. 
Zhang, R. P., \& Ji, Q. F. (2014). Effects of low temperature stress on seed germination of japonica giant embryo rice Xijue 1. Journal of Xichang University (Natural Science Edition), 28(4), 6-7.

Zhang, Y. H. (2010). Study on the biological characteristics and nutrient components of a new giant embryo rice line. Fujian Agricultural and Forestry University.

Zhou et al. (2015). Factors affecting head rice yield and chalkiness in indica rice. Field Crops Research, 172, $1-10$.

\section{Copyrights}

Copyright for this article is retained by the author(s), with first publication rights granted to the journal.

This is an open-access article distributed under the terms and conditions of the Creative Commons Attribution license (http://creativecommons.org/licenses/by/4.0/). 